Original paper

\title{
Retrospective comparison of rectal toxicity between carbon-ion radiotherapy and intensity-modulated radiation therapy based on treatment plan, normal tissue complication probability model, and clinical outcomes in prostate cancer
}

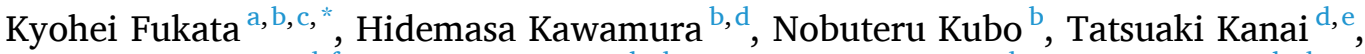 \\ Masami Torikoshi ${ }^{\text {d,f }}$, Takashi Nakano ${ }^{\text {b,d,g }}$, Mutsumi Tashiro ${ }^{\mathrm{d}}$, Tatsuya Ohno ${ }^{\text {b,d }}$ \\ a Cancer Center, School of Medicine, Keio University, Tokyo, Japan \\ ${ }^{\mathrm{b}}$ Department of Radiation Oncology, Gunma University Graduate School of Medicine, Gunma, Japan \\ ${ }^{c}$ Section of Medical Physics and Engineering, Kanagawa Cancer Center, Yokohama, Japan \\ d Gunma University Heavy Ion Medical Center, Gunma, Japan \\ e Osaka Heavy Ion Therapy Center, Osaka, Japan \\ ${ }^{\mathrm{f}}$ International Science and Technology Center, Nur-Sultan, Kazakhstan \\ ${ }^{g}$ Quantum Life and Medical Science Directorate, National Institutes for Quantum and Radiological Science and Technology, Chiba, Japan
}

\section{A R T I C L E I N F O}

\section{Keywords:}

Carbon-ion radiation therapy

Prostate cancer

Normal tissue complication probability

\begin{abstract}
A B S T R A C T
This retrospective study assessed the treatment planning data and clinical outcomes for 152 prostate cancer patients: 76 consecutive patients treated by carbon-ion radiation therapy and 76 consequtive patients treated by moderate hypo-fractionated intensity-modulated photon radiation therapy. These two modalities were compared using linear quadratic model equivalent doses in $2 \mathrm{~Gy}$ per fraction for rectal or rectal wall dose-volume histogram, 3.6 Gy per fraction-converted rectal dose-volume histogram, normal tissue complication probability model, and actual clinical outcomes. Carbon-ion radiation therapy was predicted to have a lower probability of rectal adverse events than intensity-modulated photon radiation therapy based on dose-volume histograms and normal tissue complication probability model. There was no difference in the clinical outcome of rectal adverse events between the two modalities compared in this study.
\end{abstract}

\section{Introduction}

Carbon-ion radiotherapy (CIRT) for the treatment of prostate cancer initially began as a clinical trial at National Institute of Radiological Science (NIRS) in $1994[1,2]$. The clinical trial results showed high local and biochemical relapse-free survival rate and a lower risk organ complication rate compared with the radiation therapy using X-ray. Subsequent reports have also demonstrated the efficacy of CIRT in prostate cancer treatment [1,3-6].

Carbon ion beams deposit energy in matter according to the characteristic Bragg peak, losing the most energy at a finite depth of their trajectories in a medium. The Bragg peak realizes the sharp distal fall-off in the irradiation field. In addition, carbon ions are heavier in mass than other particles used for radiation therapy, such as electron or proton; carbon ion beams have a smaller lateral scattering component. This results in a small divergence of the carbon ion beam. Therefore, not only can high dose conformality to the target be obtained but also the dose to the surrounding normal tissue is reduced. Carbon ion beams are characterized as high-linear energy transfer (LET) beams. They transfer a larger mean energy per unit length in the body than low-LET radiations such as X-ray, electron, or proton beams. The high-LET beam shows no degradation in its cell-killing effect on hypoxic cells or cells in a radioresistant cell cycle. Therefore, the advantage of CIRT over other radiation therapies is its sharper physical dose distribution, and the cellkilling effect is not compromised, even for types of targets that are not responsive to other radiation sources. At present, most patients with prostate cancer receive photon radiotherapy. Intensity-modulated radiotherapy (IMRT) is a representative technique in photon radiotherapy that utilizes inverse planning and intensity modulation. The optimization process enables the delivery of a highly conformal dose to

\footnotetext{
* Corresponding author at: Cancer Center, School of Medicine, Keio University, 35 Shinanomachi, Shinjuku-ku, Tokyo, Japan.

E-mail address: kfukata@rad.med.keio.ac.jp (K. Fukata).
} 
the target, while sparing the normal tissue. Recently, it has been shown that the alpha/beta ratio (parameter from linear quadratic (LQ) model [7]) of prostate cancer is small [8]. Hypofractionated irradiation has been used to take advantage of this; as a result, non-inferiority in tumor control and fewer rectal adverse events compared to conventional fractionated irradiation have been reported [9-11].

In the present study, we investigated whether CIRT or IMRT is associated with fewer rectal adverse events in prostate cancer treatment. In the facility where this study was conducted, both the CIRT and IMRT modalities are available for patients with prostate cancer $[6,12]$. Because the same oncologist groups work at both facilities, the contouring definition is the same. It also allows for the assessment of the grade of the side effects from the same perspective.

In this study, rectal dose-volume histograms (DVHs) of CIRT and IMRT using 76 patient plans each was retrospectively compared. The probability of rectal toxicity was calculated using the Lyman-Kutcher-Burman (LKB) NTCP model and was compared with the clinical outcomes.

\section{Materials and Methods}

\section{Patient selection}

This study retrospectively analyzed data from 152 patients obtained at Gunma University Hospital from 2010 to 2013: 76 consecutive patients treated by CIRT and 76 consecutive patients treated by IMRT. Exclusion criteria were patients with a history of pelvic or prostate radiation therapy or the presence of concurrent active malignancies or inflammatory disease. Details of the patient backgrounds are presented in Table 1. Gastrointestinal toxicity was scored using the Common Terminology Criteria for Adverse Events (CTCAE), version 4.0. This study was approved by the Institutional Review Board of Gunma University Hospital, Gunma, Japan, under referral numbers 1308 for IMRT and HS2019-130 for CIRT as an opt-out consent model.

\section{Contouring}

Computed tomography (CT) images were acquired using Aquilion LB (Self-Propelled, Toshiba Medical Systems, Tokyo, Japan) for CIRT and LightSpeed RT16 (GE Healthcare, Chicago, Illinois, United States) for IMRT. Contouring was performed by a radiation oncologist and reviewed by a radiation oncologist who specialized in genitourinary tumors. Rectal contouring was defined from the rectosigmoid flexure to the anus. The same rectal contouring definition was used for both modalities. The rectal volumes of the two modalities were subjected to Shapiro-Wilk test (SW) and Mann-Whitney $U$ test (MW-U) to test the difference.

\section{Treatment planning}

\section{Carbon-ion radiation therapy}

Irradiation was performed by a carbon-ion beam at Gunma University Heavy Ion Medical Center. The dosimetric system of Gy (relative biological effectiveness [RBE]) proposed by Kanai et al. was used [13]. The RBE model used in this study is known as the "mixed-beam model," which is a clinically used RBE model for CIRT in Japan. This model is based on in vivo experiments in human salivary gland (HSG) cells. The ridge filter was designed to produce a flat survival fraction of HSG cells in the spread-out Bragg peak. The LET and RBE of neutron therapy performed at NIRS were compared to those in a typical CIRT field. The RBE of CIRT was determined by searching the position that has the same LET as that of neutron beams. In Japan, a microdosimetric kinetic model (MKM) is also used clinically [14]. In European countries, the local effect model (LEM) is mainly used [15]. The difference between these RBE models is still under discussion [16]. The treatment plan was calculated by the XiO-N treatment planning system (TPS) (Mitsubishi Electric,
Table 1

Patient background, prescription, and irradiation method.

\begin{tabular}{|c|c|c|c|}
\hline Modality & & CIRT & IMRT \\
\hline Treatment period & & 2010-2011 & 2010-2013 \\
\hline Number of patients & & 76 & 76 \\
\hline Age in years (median, range) & & $66(53-88)$ & $\begin{array}{l}67.5 \\
(45.6-88.0)\end{array}$ \\
\hline $\begin{array}{l}\text { Follow-up in month } \\
\text { (median, range) }\end{array}$ & & $51(8-58)$ & $\begin{array}{l}29.1 \\
(12.6-53.8)\end{array}$ \\
\hline \multicolumn{4}{|l|}{ Tumor Stage } \\
\hline & 1 & 23 & 22 \\
\hline & 2 & 26 & 30 \\
\hline & 3a & 24 & 17 \\
\hline & $3 b$ & 2 & 6 \\
\hline & 4 & 1 & 1 \\
\hline \multicolumn{4}{|l|}{ Gleason Score } \\
\hline & $5-6$ & 4 & 2 \\
\hline & 7 & 42 & 47 \\
\hline & $8-10$ & 30 & 27 \\
\hline \multicolumn{4}{|l|}{ iPSA (ng/ml) } \\
\hline & $\leqq 10$ & 41 & 42 \\
\hline & $10-20$ & 16 & 21 \\
\hline & $>20$ & 19 & 13 \\
\hline \multicolumn{4}{|l|}{ Risk levels* } \\
\hline & Low & 3 & 1 \\
\hline & Intermediate & 30 & 34 \\
\hline & High & 43 & 41 \\
\hline \multicolumn{4}{|l|}{$\begin{array}{l}\text { Neoadjuvant hormonal } \\
\text { therapy }\end{array}$} \\
\hline & Yes & 69 & 74 \\
\hline & No & 7 & 2 \\
\hline \multicolumn{4}{|l|}{ Prescription } \\
\hline & Dose & 57.6 Gy (RBE) & $63 \mathrm{~Gy}$ \\
\hline & Normalization & $* *$ & PTV D95\% \\
\hline & Fraction & 16 & 21 \\
\hline Irradiation Method & Broad beam & $\begin{array}{l}\text { Fixed field } \\
\text { IMRT }\end{array}$ & \\
\hline Port \# & $* * *$ & 7 & \\
\hline Port per day & 1 & 7 & \\
\hline
\end{tabular}

"If tumor size between T1c and T2bNOM0 and iPSA $<10 \mathrm{ng} / \mathrm{ml}$ and Gleason Score $\leq 6$, low-risk. If tumor size larger than T3 and iPSA $\geq 20 \mathrm{ng} / \mathrm{ml}$ or Gleason Score $\geq 8$, high-risk. Else, intermediate-risk group.

**32.4 Gy (RBE) for PTV1, 25.2 Gy (RBE) for PTV2 to iso-center.

$* * * 3$ for PTV1, 2 for PTV2

CIRT, carbon-ion radiotherapy; IMRT, intensity-modulated radiotherapy; iPSA, initial prostate-specific antigen; PTV, planning target volume; RBE, relative biological effectiveness.

Tokyo, Japan). The dose grid size was $2 \times 2 \times 2 \mathrm{~mm}^{3}$. The treatment procedure was divided into two sequences, namely irradiation to PTV1 and PTV2. For the contouring of PTV1, margins of $10 \mathrm{~mm}$ for anterior, left, and right, $6 \mathrm{~mm}$ for superior and inferior, $5 \mathrm{~mm}$ for posterior were added to the prostate and proximal seminal vesicle. For PTV2, margins of $10 \mathrm{~mm}$ for anterior, left, and right were added to the prostate and proximal seminal vesicle, and the posterior side was cut horizontally at the anterior wall of the rectum. In the first sequence, $32.4 \mathrm{~Gy}$ (RBE) in nine fractions was delivered to PTV1 using the beams from anterior, left, and right direction. In the second sequence, 25.2 Gy (RBE) in seven fractions was delivered to PTV2 using the beams from left and right direction. A total dose of $57.6 \mathrm{~Gy}$ (RBE) in 16 fractions was prescribed. The irradiation schedule was 4 days/week: Tuesday, Wednesday, Thursday, and Friday. Prescription point was set to the iso-center. The treatment plan was intended to encompass the PTV with $95 \%$ of the prescribed isodose line if a rectal dose is acceptable to the criteria. For the rectum, the treatment plan was designed so that DVH would not exceed $24 \%, 12 \%$, and $6 \%$ of V50\%, V70\%, and V90\%, respectively, of the prescribed dose. The patient was fixated using immobilization shell that was made of a thick thermoplastic around the abdomen. Twodimensional orthogonal X-ray images (anterior-posterior and left-right) were used for the patient setup with bony anatomy matching. In this method, the position of the prostate itself cannot be determined. Therefore, the inter and intra-fractional motions remain as setup errors. 
Intensity-modulated radiotherapy

Irradiation was performed using 7-fixed field dynamic IMRT by Trilogy (Varian Medical Systems, USA), and the TPS was Eclipse, version 8.0 (Varian). The dose grid size was $2 \times 2 \times 2 \mathrm{~mm}^{3}$. Prescription dose was $63 \mathrm{~Gy}$ to $95 \%$ of the PTV in 21 fractions. The following margins were added to the prostate and proximal seminal vesicle to define PTV: $4 \mathrm{~mm}$ for posterior and $7 \mathrm{~mm}$ for the other five directions. The irradiation schedule was 3 days/week: Monday, Wednesday, and Friday. The inverse planning method was performed to optimize the dose distribution to meet the following dose constraints. The maximum dose should be $<107 \%$ of the prescribed dose, and V55 Gy and V20 Gy of the rectum should be lower than $14 \%$ and $60 \%$, respectively. No immobilization device was used for the patient. A cone-beam CT image was acquired for every treatment for the patient setup, and prostate matching was also used.

\section{Dose-volume histogram (DVH) comparison}

Dose distribution was exported to a Digital Imaging and Communications in Medicine (DICOM) RT file with the DVH from both treatment planning systems. The DICOM RT files were analyzed using an in-house program that was coded using Python [17]. All the DVHs had $0.1 \mathrm{cGy}$ bin size. The extracted DVH data were compared using the following three steps.

First, both modalities were compared using their dose axis converted to an LQ equivalent dose in 2 Gy fraction (EQD2) [7]. The equation for EQD2 was as follows:

$\mathrm{EQD} 2=D \cdot\left(\frac{\alpha / \beta+D / N}{\alpha / \beta+2}\right)$

where $\mathrm{D}$ is the total dose, $\mathrm{N}$ is the number of fractions of the treatment. Akimoto et al. and Huang et al. reported the rectal toxicity tolerance line in their study $[18,19]$. Therefore, these tolerance lines were converted to EQD2 and compared with the rectal DVH in this study. An alpha/beta ratio of 3 Gy was used for this comparison referring to the Quantitative Analyses of Normal Tissue Effects in the Clinic report [20]. The EQD2converted DVHs were compared for PTVs. An alpha/beta ratio of 2.48 Gy, which was calculated by Walsh et al [21], was used. Because it is not obvious whether converting the CIRT dose to EQD2 is appropriate, the following procedure was investigated in the next step.

Second, because the EQD conversion of photon radiation therapy is well-discussed [22-24], the IMRT DVH was converted to a LQ equivalent dose in $3.6 \mathrm{~Gy}$ (EQD $3.6 \mathrm{~Gy}$ ), whereas the CIRT dose was left unchanged.

Third, the DVH of the rectal wall having a thickness of $3 \mathrm{~mm}$ was compared with the multi-modality planning study conducted by Georg et al. [25]. The EQD2 conversion was also used in this comparison.

Statistical tests were performed on the three comparisons described above. The DVHs were linearly interpolated in $1 \mathrm{~Gy}$ increments from 0 to 80 Gy. These interpolated DVHs were tested using the following procedure:

First, SW was performed to check if there was normality in both groups. The MW-U was performed if either did not show normality. If both groups were normally distributed, the F-test was performed to see if their populations have the same variance. If they have the same variance, Student's $t$-test was performed. If the variances are not equal, Welch's $t$-test was performed. A significance level of $5 \%$ was used for all statistical tests.

\section{NTCP calculation}

The LKB NTCP model was calculated for both modalities [26-28]. Generalized equivalent uniformed dose (gEUD) was employed in the calculation [29] as follows:

$\mathrm{NTCP}=\frac{1}{\sqrt{2 \pi}} \int_{-\infty}^{\mathrm{t}} e^{-x^{2} / 2} d x$
$\mathrm{t}=\frac{\mathrm{gEUD}-\mathrm{TD}_{50}}{\mathrm{~m} \cdot \mathrm{TD}_{50}}$

$\operatorname{gEUD}=\left(\sum_{\mathrm{i}} v_{\mathrm{i}} D_{\mathrm{i}}^{\frac{1}{\mathrm{n}}}\right)^{\mathrm{n}}$

where $\mathrm{n}, \mathrm{m}, \mathrm{TD}_{50}$ are LKB NTCP parameters. The $\mathrm{n}$ parameter represents the volume effect, i.e., the seriality or parallelism of the organs. The $m$ parameter corresponds to the gradient of the NTCP curve. The $\mathrm{TD}_{50}$ parameter is the dose that has a $50 \%$ probability of causing complications when the organ is uniformly irradiated with $\mathrm{TD}_{50} \cdot \mathrm{v}_{\mathrm{i}}$ and $\mathrm{D}_{\mathrm{i}}$ are volume bin and dose of DVH, respectively. $\mathrm{x}$ is an arbitrary real number that aims to integrate a Gaussian distribution in equation (2). The NTCP parameters used in this study are enlisted in Table 2 ([20,30-34]).). The EQD2 conversion was also used in this NTCP calculation. In the EQD2 conversion, an alpha/beta ratio of $3 \mathrm{~Gy}$ (Michalski et al. [20]) and 5.4 Gy (Brenner et al. [35]) were used.

\section{Results}

The mean and standard deviation of rectal volumes were 62.01 (14.94) cc (SW: $p<0.01$ ) and 59.25 (17.51) cc (SW: $p<0.01$ ) for CIRT and IMRT, respectively. There was no significant difference in the mean rectal volume between the two groups (MW-U: $p=0.155$ ).

\section{DVH comparison}

In all three rectal DVH comparisons, there was a significant difference between the means of CIRT and IMRT groups for all bins except for the 0-1 Gy bin. For the EQD2, and EQD3.6 comparison, MW-U was used for all bins. In the comparison of rectal wall DVH, Welch's $t$-test was used for the 60-63 Gy dose bin; however, MW-U was used for all other bins.

DVHs converted to EQD2 using an alpha/beta ratio of $3 \mathrm{~Gy}$ for rectal DVH and $2.48 \mathrm{~Gy}$ for PTVs are shown in Fig. 1. Rectal DVH of CIRT was significantly lower than that of IMRT (MW-U: $p<0.01$ ). The mean and standard deviation of D95\% of EQD2 for PTV for IMRT, PTV1, and PTV2 for CIRT was 77.21 (0.18) Gy, 63.99 (3.11) Gy (RBE), and 76.47 (1.02) Gy (RBE). These doses correspond to $100 \%, 88.1 \%$, and $98.6 \%$ of the prescribed dose, respectively. In Fig. 1, the reference line reported by Akimoto et al. [18] and Huang et al. [19] were plotted together. The data points on the publications were also converted to EQD2 using an alpha/beta ratio of $3 \mathrm{~Gy}$. Here, both CIRT and IMRT show lower rectal doses than the reported dose thresholds for rectal toxicity grade of over 2.

In Fig. 2, CIRT DVH and EQD3.6 Gy-converted IMRT DVH were plotted. The statistical test showed significant differences for all dose bins except $0-1$ Gy bin in the figure (MW-U: $p<0.01$ ). In this comparison, CIRT DVH also showed a significantly lower rectal DVH than that shown for IMRT DVH.

Fig. 3 shows the mean rectal wall DVH of CIRT and IMRT. Both modalities used EQD2 conversion for their dose bin. The rectal wall dose was significantly lower with CIRT than with IMRT (Welch's $t$-test for 60 Gy, 62 Gy bin: $p<0.01$, MW-U for other: $p<0.01$ ). The data points reported by Georg et al. were shown in double-quotes in the figure legends [25]. The CIRT showed lower DVH than "Carbons" and IMRT showed lower DVH than "VMAT," although these pairs (CIRT/"Carbons" and DVH/"VMAT") are the same modalities.

\section{NTCP calculation}

The predicted NTCPs using several models ([20,30-34]) is summarized in Table 2. In the calculation of rectal toxicity grade $>2$, the predicted NTCP range was $0.00 \%-1.67 \%$ and $0.03 \%-4.41 \%$ for CIRT and IMRT, respectively. In rectal toxicity grade $>1$, the predicted NTCP 
Table 2

Mean NTCP for CIRT and IMRT and the parameters used for the calculation for each of the 76 consecutive patients*.

\begin{tabular}{|c|c|c|c|c|c|c|c|}
\hline Author & Endpoint & $\mathrm{N}$ & $\mathrm{m}$ & TD50 & $\alpha / \beta$ for EQD2 & CIRT NTCP & IMRT NTCP \\
\hline Fukahori [30] & Grade over 2 RTOG & 0.012 & 0.046 & 69.1 & - & $0 \% \pm 0 \%$ & \\
\hline \multirow[t]{2}{*}{ Michalski [20] } & Grade over 2 rectal toxicity & 0.09 & 0.13 & 76.9 & 3 & $0.85 \% \pm 0.40 \%$ & $2.85 \% \pm 1.60 \%$ \\
\hline & & & & & 5.4 & $0.28 \% \pm 0.14 \%$ & $1.41 \% \pm 0.81 \%$ \\
\hline \multirow[t]{2}{*}{ Tucker [31] } & RTOG grade over 2 & 0.08 & 0.14 & 78 & 3 & $1.64 \% \pm 0.62 \%$ & $4.35 \% \pm 1.98 \%$ \\
\hline & & & & & 5.4 & $0.63 \% \pm 0.24 \%$ & $2.34 \% \pm 1.09 \%$ \\
\hline \multirow[t]{2}{*}{ Sohn [32] } & Grade over 2 CTCAE3.0 & 0.08 & 0.108 & 78.4 & 3 & $0.27 \% \pm 0.16 \%$ & $1.32 \% \pm 0.94 \%$ \\
\hline & & & & & 5.4 & $0.06 \% \pm 0.04 \%$ & $0.49 \% \pm 0.37 \%$ \\
\hline \multirow[t]{4}{*}{ Rancati [33] } & Grade over 2 bleeding & 0.23 & 0.19 & 81.9 & 3 & $0.15 \% \pm 0.08 \%$ & $0.58 \% \pm 0.39 \%$ \\
\hline & & & & & 5.4 & $0.09 \% \pm 0.05 \%$ & $0.41 \% \pm 0.27 \%$ \\
\hline & & 0.24 & 0.14 & 75.7 & 3 & $0.01 \pm 0.01 \%$ & $0.09 \% \pm 0.12 \%$ \\
\hline & & & & & 5.4 & $0.00 \% \pm 0.00 \%$ & $0.05 \% \pm 0.07 \%$ \\
\hline Fukahori [30] & Grade over 1 RTOG & 0.035 & 0.1 & 63.6 & - & $1.22 \% \pm 0.37 \%$ & \\
\hline \multirow[t]{2}{*}{ Gulliford [34] } & Grade over 1 & 0.14 & 0.26 & 59.2 & 3 & $18.19 \% \pm 3.67 \%$ & $28.81 \% \pm 6.95 \%$ \\
\hline & & & & & 5.4 & $13.36 \% \pm 2.73 \%$ & $23.81 \% \pm 5.83 \%$ \\
\hline
\end{tabular}

*The DVH was calculated for both modalities. The LKB NTCP parameter reported by Fukahori et al. [30] was derived from the result of the CIRT in NIRS. Other parameters were estimated by clinical outcome of photon radiotherapy. The NTCPs using parameters of photon radiotherapy were calculated using EQD2 with an alpha/beta ratio of $3 \mathrm{~Gy}$ and $5.4 \mathrm{~Gy}$, respectively. A statistically significant difference was observed in all pairs of CIRT NTCP and IMRT NTCP $(p<0.05$ by the Mann-Whitney $U$ test).

CTCAE, Common Terminology Criteria for Adverse Events; CIRT, carbon-ion radiotherapy; DVH, dose-volume histogram; EQD2, equivalent dose in 2 Gy fraction; IMRT, intensity-modulated radiotherapy; LKB, Lyman-Kutcher-Burman; NTCP, normal tissue complication probability; RTOG, Radiation Therapy Oncology Group.

(a)

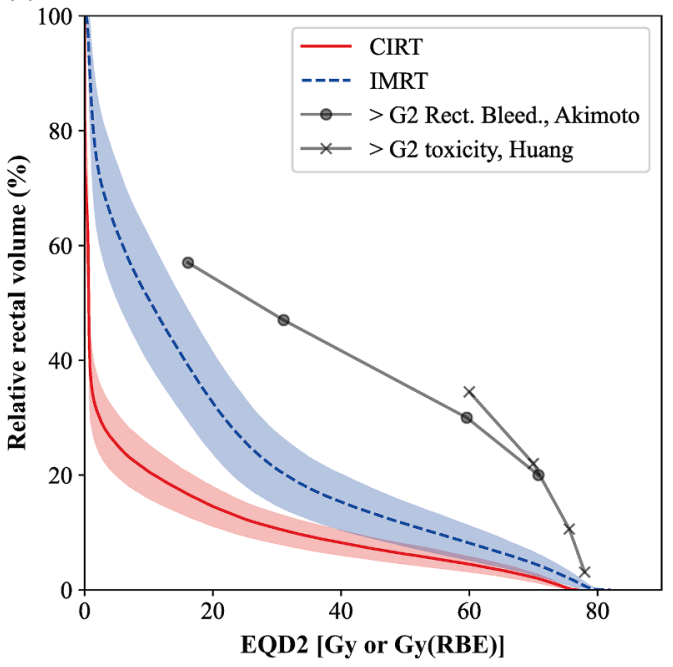

(b)

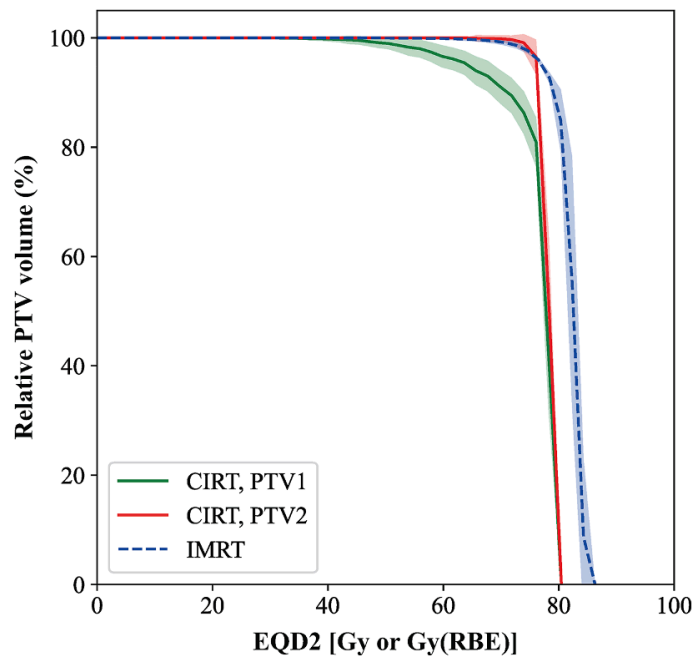

Fig. 1. Relative rectal DVH using LQ equivalent dose in $2 \mathrm{~Gy}$ per fraction. Mean DVHs plus/minus standard deviation were plotted. Circle and cross mark data points show the threshold value of rectal toxicity grade $>2$ reported by Akimoto et al. [18] and Huang et al. [19], respectively. CIRT, carbon-ion radiotherapy; DVH, dose-volume histogram; IMRT, intensity-modulated radiotherapy; LQ, linear quadratic.

range was $1.25 \%-18.5 \%$ and $24.4 \%-29.7 \%$ for CIRT and IMRT, respectively. The predicted NTCP was lower for CIRT than for IMRT for both grades and all parameters.

Predicted NTCP and clinical outcome were compared with the data from other studies, as shown in Table 3 ([1,3-5,11,36-39]). Gastrointestinal toxicities for CIRT were between the estimation by NTCP models for both grades. Gastrointestinal toxicity grade $>1$ for IMRT was overestimated, while a grade $>2$ was within the NTCP estimation.

\section{Discussion}

In this study, the rectal DVH in CIRT was lower than that for IMRT in all the comparisons performed. In the comparison using EQD2-DVH, both CIRT and IMRT were under the threshold of G2 rectal toxicity reported in the other studies $[18,19]$. In the comparison using rectal wall EQD2-DVH, the DVHs of CIRT and IMRT were lower than those reported in the study by Georg et al. using "carbon" and "VMAT," respectively, despite these respective pairs being the same modalities. This result arises mainly from the differences in the definition of the PTV and optimization strategy. In fact, the CIRT in this study uses the 2-step method with PTV1 and PTV2, while carbon uses a single PTV. PTV2 results in a large reduction in rectal dose because the PTV2 margin behind the rectum is zero. The PTV margins used for IMRT in this study are $1 \mathrm{~mm}$ smaller in the superior-inferior and posterior directions than those used for VMAT. Besides, the rectal dose constraint used in the optimization of IMRT is strictly set and followed. These may be the main reasons that contribute to explain why the rectal DVH of CIRT and IMRT are lower than those of carbon and VMAT. The study by Georg et al. is a hypothetical planning study, but the present study differs in that it uses the actual plan used in the treatment.

The NTCPs were evaluated using multiple parameters. The calculation using the following situations were performed: absolute dose or EQD2, using the alpha/beta ratio of $3.0 \mathrm{~Gy}$ or $5.4 \mathrm{~Gy}$, using the NTCP parameter for IMRT or CIRT. Regardless of the situations used for the calculation, CIRT showed lower estimated NTCP of rectal toxicity to a greater degree than IMRT.

As summarized in Table 3, rectal toxicity in CIRT has been reported by Ishikawa, Okada, Nomiya, and Takakusagi et al $[1,3-5]$. In all these 


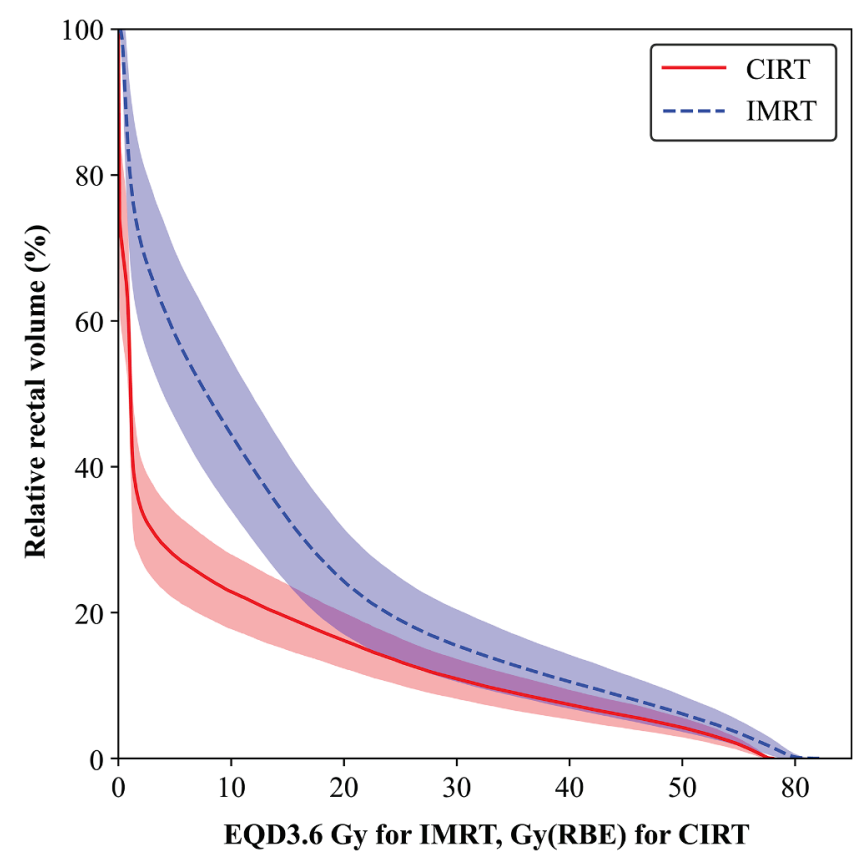

Fig. 2. Mean rectal DVH for CIRT and IMRT. For IMRT, DVH was converted to linear quadratic equivalent dose in $3.6 \mathrm{~Gy}$ per fraction. CIRT, carbon-ion radiotherapy; DVH, dose-volume histogram; IMRT, intensity-modulated radiotherapy.

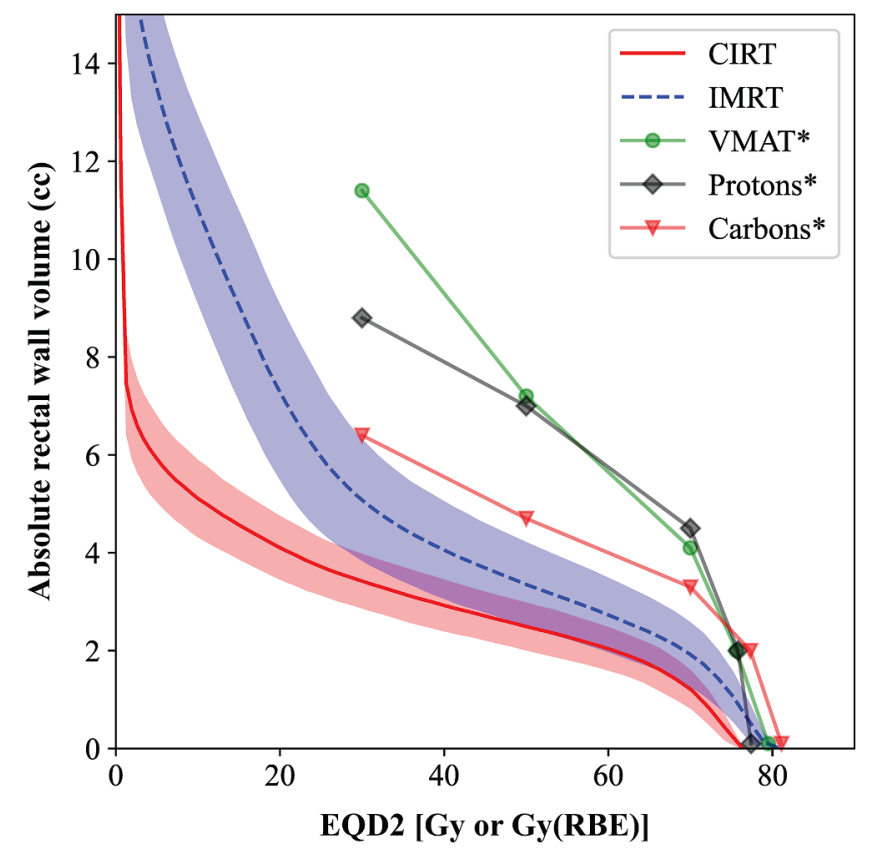

Fig. 3. Absolute rectal wall ( $3 \mathrm{~mm}$ ) DVH using linear quadratic equivalent dose in 2 Gy per fraction. Mean DVHs plus/minus standard deviation were plotted. Data points with an asterisk were published data by Georg et al. [25].CIRT, carbon-ion radiotherapy; DVH, dose-volume histogram; HDR, high dose rate; IMRT, intensity-modulated radiotherapy; LDR, low-dose-rate; VMAT, volumetric modulated arc therapy.

reports, rectal toxicities of grade 2 or higher ranged from $0 \%$ to $2 \%$, and those of grade 1 or higher ranged from $4.7 \%$ to $9.6 \%$. The clinical outcomes obtained in this study for CIRT (1.3\% and $9.2 \%$, respectively) were within the range of those reports. The number of patients having actual rectal toxicities as clinical outcome grade of over 1 and 2 were identical for IMRT and CIRT, respectively. Based on models such as DVH and NTCP, CIRT was expected to have a lower frequency of rectal adverse events than IMRT, but there was no difference between CIRT and IMRT in clinical outcomes.

\section{Effect of moderate hypofractionation in IMRT}

Moderate hypofractionation was used in IMRT - 3 Gy per fraction. Several reports used a moderate hypofractionation irradiation schedule. Akimoto et al. [18] and Patel et al. [40] showed the rectal toxicity rate in three-dimensional conformal radiation therapy using irradiation schedules of $69 \mathrm{~Gy}$ in 23 fractions and $66 \mathrm{~Gy}$ in 22 fractions. The rates of rectal toxicity grade $>2$ was $25 \%$ and $27 \%$ for the 69 Gy and $66 \mathrm{~Gy}$ groups, respectively. These values for the rates of rectal toxicity are higher than those reported for IMRT, as listed in Table 3. In other words, rectal toxicity is reduced when a hypofractionated irradiation schedule is used along with IMRT. For instance, Lieng et al. [11] showed the results of rectal adverse events of IMRT with $66 \mathrm{~Gy}$ in 23 fractions. This fractionation scheme is the same as that used by Patel et al. [40] The study conducted by Lieng et al. was a Phase II clinical trial, and the 66 $\mathrm{Gy} / 22$ fractions arm, which had more adverse events, was terminated in the middle of the trial. The other arm of the clinical trial conducted by Lieng et al. (60 Gy/20 fractions) had $4.2 \%$ of rectal adverse events of grade 2 or higher, which is lower than those reported for other IMRT studies, as shown in Table 3. The irradiation schedule used in the present study was $63 \mathrm{~Gy} / 21$ and adopted the fractionation scheme just between the two arms of the study by Lieng et al. From these results, we can conclude that IMRT is essential to reduce the rectal adverse events. In contrast, the study by Hoffman et al. [37] reported a rectal adverse event rate of $5 \%$ due to irradiation at $75.6 \mathrm{~Gy} / 42$ fractions, which is comparable to that reported by Lieng et al. This is classified as hyperfractionated irradiation. Therefore, it is not possible to conclude whether hypofractionation is essential to reduce rectal adverse events from the data in this Table.

The definition of the PTV is also an important factor that affects the rectal dose.

As shown in Table 3, with respect to the posterior margin, it was observed that Kupelian et al. [38] used the same posterior margin as the present study (4 mm), followed by Hoffman et al. (4-8 mm), Spratt et al. [36] and Takemoto et al. [39] (6 mm), and Lieng et al. (7 mm). We found that a smaller PTV margin in the posterior direction did not necessarily result in lower rectal adverse events.

The present study showed the lowest grade $>2$ rectal toxicity (1.3\%) compared with those observed in other studies, as shown in Table 3. These differences among studies may be attributed to the definitions of the PTV and prescription (by prescription of the dose to the point or by volumetric prescription). Furthermore, as mentioned in the Methods section herein, the present study used a characteristic irradiation schedule (Monday, Wednesday, and Friday irradiation), but the schedule was the same as that used by Akimoto et al. [18] Whether this characteristic irradiation schedule reduced the rectal toxicity requires a more detailed investigation.

\section{Study limitations}

The calculated NTCP of gastrointestinal toxicity grade $>1$ for IMRT was larger than the clinical outcome. In this study, endoscopic examinations were only performed when patients complained of disturbances. The number of rectal adverse events collected by this method may be lower than those collected by endoscopic examination at each follow-up. The median follow-up period of IMRT ( 29 months) was relatively short compared with that of CIRT (51 months). In this study, rectal adverse events of grade $>1$ in IMRT occurred between 12.6 and 53.8 months. In a study by Patel et al. [40], who used the same dose per fraction as the present study with a median follow-up of 90 months, rectal adverse events of grade $>2$ were confirmed after 36 months. In CIRT, according to Ishikawa et al., whose study included a median follow-up of 46 
Table 3

NTCP Model Calculation and Clinical Outcome of Gastrointestinal Toxicity Compared With Other Studies.

\begin{tabular}{|c|c|c|c|c|c|c|c|c|}
\hline Authors & Year & Treatment & No. of PTs & Dose & Fractions & $>$ Grade $2(\%)$ & $>$ Grade1 (\%) & Criteria \\
\hline Kupelian & 2005 & IMRT & 100 & $70 \mathrm{~Gy}$ & 28 & 11.0 & & RTOG \\
\hline Spratt & 2013 & IMRT & 1002 & $86.4 \mathrm{~Gy}$ & 48 & 4.4 & & CTCAE 4.0 \\
\hline \multirow[t]{2}{*}{ Hoffman } & 2014 & IMRT & 101 & $75.6 \mathrm{~Gy}$ & 42 & 5.0 & 21.8 & RTOG \\
\hline & & IMRT & 102 & $72 \mathrm{~Gy}$ & 30 & 10.8 & 37.3 & RTOG \\
\hline \multirow[t]{2}{*}{ Lieng } & 2017 & IMRT & 96 & $60 \mathrm{~Gy}$ & 20 & 4.2 & & RTOG \\
\hline & & & 27 & $66 \mathrm{~Gy}$ & 22 & 18.5 & & RTOG \\
\hline \multirow[t]{3}{*}{ Takemoto } & 2018 & IMRT & 74 & 74/78 Gy & $37 / 39$ & 12.4 & & CTCAE 4.0 \\
\hline & & & 101 & 73.5/77.7 Gy & $35 / 37$ & 14.1 & & CTCAE 4.0 \\
\hline & & & 173 & 72.6/74.8 Gy & $33 / 34$ & 7.9 & & CTCAE 4.0 \\
\hline Ishikawa & 2012 & CIRT & 927 & 57.6-66.0 Gy (RBE) & $16-20$ & 1.9 & & RTOG \\
\hline Okada & 2012 & CIRT & 198 & 57.6 Gy (RBE) & 16 & 1.5 & 9.6 & RTOG \\
\hline Nomiya & 2014 & CIRT & 46 & 51.6 Gy(RBE) & 12 & 0 & 7.0 & CTCAE 4.0 \\
\hline Takakusagi & 2020 & CIRT & 253 & 51.6 Gy(RBE) & 12 & 1.2 & 4.7 & CTCAE 4.0 \\
\hline \multicolumn{9}{|l|}{ Present study } \\
\hline \multirow[t]{2}{*}{ Estimated NTCP } & & IMRT & 76 & $63 \mathrm{~Gy}$ & 21 & $0.05-4.35$ & $23.81-28.81$ & CTCAE 4.0 \\
\hline & & CIRT & 76 & $57.6 \mathrm{~Gy}(\mathrm{RBE})$ & 16 & $0.0-1.64$ & $1.22-18.19$ & CTCAE 4.0 \\
\hline \multirow[t]{2}{*}{ Clinical Outcome } & & IMRT & 76 & $63 \mathrm{~Gy}$ & 21 & 1.3 & 9.2 & CTCAE 4.0 \\
\hline & & CIRT & 76 & 57.6 Gy(RBE) & 16 & 1.3 & 9.2 & CTCAE 4.0 \\
\hline
\end{tabular}

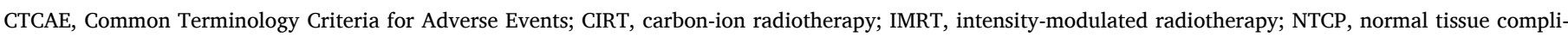
cation probability; RTOG, Radiation Therapy Oncology Group.

months, the last patient reported rectal toxicity of grade $>2$ approximately three years after the treatment [41]. Additionally, according to Nomiya et al., whose study included a median follow-up of 29 months, the last patient reported rectal toxicity of grade $>2$ approximately 40 months after the treatment [42]. In the case of CIRT, most adverse events occur between 6 months and two years. Therefore, the number of rectal adverse events of IMRT in this study may increase if the follow-up period is identical to that of CIRT.

This study was performed under the assumption that the alpha/beta value of the LQ model evaluated in photon radiotherapy was directly applicable to the CIRT for EQD2 conversion. However, there is no evidence that the alpha/beta value calculated by photon radiotherapy can be used for CIRT because alpha and beta are functions with depth in the carbon ion beam. In the TPS of CIRT, the non-flatness of alpha and beta were considered in the design of the ridge filter to deliver a flat biological dose to the spread-out Bragg peak. Therefore, the alpha/beta value in the irradiation field cannot be represented by a single value in CIRT. Furthermore, one port/day irradiation was used in CIRT. The beam path differs day by day; this fluctuation will result in a different biological effect compared with irradiation by all ports/day. Therefore, the use of the alpha/beta value calculated by photon radiotherapy directly in CIRT is less evidenced. Nevertheless, no alternative method other than this approach is available at present for fractionation conversion for CIRT, and this study was therefore based on this hypothesis.

As mentioned above, we directly used the alpha/beta value calculated by photon radiotherapy for the EQD2 conversion of CIRT in this study. Moreover, we focused on tumor control. According to studies conducted by Kawamura et al. and Kubo et al., the 5-year biochemical relapse-free rate was $92.7 \%$ for CIRT and $92.1 \%$ for IMRT $[6,12]$. For the comparison, we converted the prescribed dose to a biological effective dose (BED). We used the alpha/beta ratio of prostate cancer as $2.48 \mathrm{~Gy}$ calculated by Walsh et al. [21]. The BED was calculated as $141.1 \mathrm{~Gy}$ (RBE) for CIRT and 139.2 Gy for IMRT. The difference in the BEDs for the two modalities was $1.43 \%$. The 5 -year biochemical relapse-free rate was comparable, and the BEDs calculated based on the LQ model were also comparable. This result does not deny the use of the EQD2/BED conversion in CIRT.

In the present study, the alpha/beta value of prostate cancer was set at $2.48 \mathrm{~Gy}$, based on the study by Walsh et al [21]. However, as shown below, there is a huge controversy regarding the alpha/beta value of prostate cancer. According to the paper published by Brenner et al. in 1999 [43], the alpha/beta value of prostate cancer is $1.5 \mathrm{~Gy}$. In 2012, Oliveira et al. published that the alpha/beta value is approximately 2.7 Gy [44]. In a paper published by Cosset et al. [45] in 2019, three studies published in 2018 on the alpha/beta value of prostate cancer had a range of 2.7-4.9 Gy. There has been much discussion over the past 20 years about the alpha/beta ratio of prostate cancer. Indeed, it is subject to a large uncertainty.

The NTCP parameter calculated by Fukahori et al. used several dose and fractionation schedules without considering the fractionation factor as follows: $57.6 \mathrm{~Gy}$ (RBE) in 16 fractions, $60 \mathrm{~Gy}$ (RBE) in 20 fractions, 63 Gy (RBE) in 20 fractions, 66 Gy (RBE) in 20 fractions, and 72 Gy (RBE) in 20 fractions. Because the present study used $57.6 \mathrm{~Gy}$ (RBE) in 16 fractions, there is uncertainty with respect to the calculated NTCP that is inherent to this fractional deviation.

\section{Conclusions}

The rectal dose and toxicity for CIRT and IMRT were evaluated using DVH, the NTCP model, and clinical outcomes. CIRT showed a significantly lower rectal dose compared with IMRT in all DVH analyses in this study. In addition, the NTCP value calculated using several parameters is also significantly lower for CIRT compared with IMRT. However, the actual clinical outcome of rectal complications was the same for both modalities.

\section{Declaration of Competing Interest}

The authors declare that they have no known competing financial interests or personal relationships that could have appeared to influence the work reported in this paper.

\section{Acknowledgements}

We are grateful for the support provied by the staffs at Gunma University Hospital and Heavy Ion Medical Center. The authors would like to thank Enago (www.enago.jp) for the English language review.

\section{Funding}

This work was supported by JSPS KAKENHI Grant Number 20 K16708 and 19 K08129. This funding source had no role in the design of this study and will not have any role during its execution, analyses, interpretation of the data, or decision to submit results. 


\section{References}

[1] Ishikawa H, Tsuji H, Kamada T, Akakura K, Suzuki H. Carbon-ion radiation therapy for prostate cancer 2012:296-305. https://doi.org/10.1111/j.14422042.2012.02961.x.

[2] Kamada T, Tsujii H, Blakely EA, Debus J, De NW, Durante M, et al. Carbon ion radiotherapy in Japan : an assessment of 20 years. Lancet Oncol 2015;16:e93-100. https://doi.org/10.1016/S1470-2045(14)70412-7.

[3] Okada T, Tsuji H, Kamada T, Akakura K, Suzuki H, Shimazaki J, et al. Carbon ion radiotherapy in advanced hypofractionated regimens for prostate cancer: From 20 to 16 fractions. Int J Radiat Oncol Biol Phys 2012;84(4):968-72. https://doi.org/ 10.1016/j.ijrobp.2012.01.072.

[4] Nomiya T, Tsuji H, Maruyama K, Toyama S, Suzuki H, Akakura K, et al. Phase I/II trial of definitive carbon ion radiotherapy for prostate cancer: Evaluation of shortening of treatment period to 3 weeks. Br J Cancer 2014;110(10):2389-95. https://doi.org/10.1038/bjc.2014.191.

[5] Takakusagi Y, Katoh H, Kano K, Anno W, Tsuchida K, Mizoguchi N, et al. Preliminary result of carbon-ion radiotherapy using the spot scanning method for prostate cancer. Radiat Oncol 2020;15(1). https://doi.org/10.1186/s13014-02001575-7.

[6] Kawamura H, Kubo N, Sato H, Mizukami T, Katoh H, Ishikawa H, et al. Moderately hypofractionated carbon ion radiotherapy for prostate cancer; A prospective observational study "gUNMA0702". BMC Cancer 2020;20(1). https://doi.org/ 10.1186/s12885-020-6570-8.

[7] Fowler JF. The linear-quadratic formula and progress in fractionated radiotherapy. Br J Radiol 1989;62(740):679-94. https://doi.org/10.1259/0007-1285-62-740679.

[8] Vogelius IR, Bentzen SM. Meta-analysis of the alpha/beta ratio for prostate cancer in the presence of an overall time factor: Bad news, good news, or no news? Int $\mathrm{J}$ Radiat Oncol Biol Phys 2013;85(1):89-94. https://doi.org/10.1016/j. ijrobp.2012.03.004.

[9] Catton CN, Lukka H, Gu C-S, Martin JM, Supiot S, Chung PWM, et al. Randomized trial of a hypofractionated radiation regimen for the treatment of localized prostate cancer. J Clin Oncol 2017;35(17):1884-90. https://doi.org/10.1200/ JCO. 2016.71.7397.

[10] Dearnaley D, Syndikus I, Mossop H, Khoo V, Birtle A, Bloomfield D, et al. Conventional versus hypofractionated high-dose intensity-modulated radiotherapy for prostate cancer: 5 -year outcomes of the randomised, non-inferiority, phase 3 CHHiP trial. Lancet Oncol 2016;17(8):1047-60. https://doi.org/10.1016/S14702045(16)30102-4.

[11] Lieng H, Pintilie M, Bayley A, Berlin A, Bristow R, Chung P, et al. Long-term outcomes of a phase II trial of moderate hypofractionated image-guided intensity modulated radiotherapy (IG-IMRT) for localized prostate cancer. Radiother Oncol 2017;122(1):93-8. https://doi.org/10.1016/j.radonc.2016.10.017.

[12] KUBO NOBUTERU, KAWAMURA HIDEMASA, OIKE TAKAHIRO, SATO HIRO, IWANAGA MOTOTARO, MIZUKAMI TATSUJI, et al. Hypofractionated intensitymodulated radiotherapy for intermediate- And high-risk prostate cancer: A retrospective study. Vivo (Brooklyn) 2019;33(4):1235-41.

[13] Kanai T, Endo M, Minohara S, Miyahara N, Koyama-ito H, Tomura H, et al. Biophysical characteristics of HIMAC clinical irradiation system for heavy-ion radiation therapy. Int J Radiat Oncol 1999;44(1):201-10. https://doi.org/ 10.1016/S0360-3016(98)00544-6.

[14] Hawkins RB. A statistical theory of cell killing by radiation of varying linear energy transfer. Radiat Res 1994;140:366-74. https://doi.org/10.2307/3579114.

[15] Scholz M, Kellerer AM, Kraft-Weyrather W, Kraft G. Computation of cell survival in heavy ion beams for therapy: The model and its approximation. Radiat Environ Biophys 1997;36(1):59-66. https://doi.org/10.1007/s004110050055.

[16] Fossati P, Matsufuji N, Kamada T, Karger CP. Radiobiological issues in prospective carbon ion therapy trials. Med Phys 2018;45:e1096-110. https://doi.org/10.1002/ mp. 12506.

[17] Van Rossum G, Drake FL. Python Tutorial. Python Softw Found 2012;42:1-136. https://doi.org/10.1111/j.1094-348X.2008.00203 7.x.

[18] Akimoto T, Muramatsu H, Takahashi M, Saito J-I, Kitamoto Y, Harashima K, et al. Rectal bleeding after hypofractionated radiotherapy for prostate cancer: Correlation between clinical and dosimetric parameters and the incidence of grade 2 or worse rectal bleeding. Int J Radiat Oncol Biol Phys 2004;60(4):1033-9. https://doi.org/10.1016/j.ijrobp.2004.07.695.

[19] Huang E, Ollack A, Evy L, Tarkschall G, Ong L, Osen I, et al. Late Rectal Toxicity : Dose - Volume Effects of Conformal Radiotherapy for. Prostate Cancer 2002;54: 1314-21.

[20] Michalski JM, Gay H, Jackson A, Tucker SL, Deasy JO. Radiation Dose-Volume Effects in Radiation-Induced Rectal Injury. Int J Radiat Oncol Biol Phys 2010;76 (3):S123-9. https://doi.org/10.1016/j.ijrobp.2009.03.078.

[21] Walsh S, Van Der Putten W. A TCP model for external beam treatment of intermediate-risk prostate cancer. Med Phys 2013;40:1-11. https://doi.org/ 10.1118/1.4790469.

[22] Withers HR, Thames HD, Peters LJ. A new isoeffect curve for change in dose per fraction. Radiother Oncol 1983;1(2):187-91. https://doi.org/10.1016/S01678140(83)80021-8.

[23] Bentzen SM, Dörr W, Gahbauer R, Howell RW, Joiner MC, Jones B, et al. Bioeffect modeling and equieffective dose concepts in radiation oncology-Terminology, quantities and units. Radiother Oncol 2012;105(2):266-8. https://doi.org/ 10.1016/j.radonc.2012.10.006.
[24] Borst GR, Ishikawa M, Nijkamp J, Hauptmann M, Shirato H, Onimaru R, et al. Radiation pneumonitis in patients treated for malignant pulmonary lesions with hypofractionated radiation therapy. Radiother Oncol 2009;91(3):307-13. https:// doi.org/10.1016/j.radonc.2009.02.003.

[25] Georg D, Hopfgartner J, Gòra J, Kuess P, Kragl G, Berger D, et al. Dosimetric Considerations to Determine the Optimal Technique for Localized Prostate Cancer Among External Photon, Proton, or Carbon-Ion Therapy and High-Dose-Rate or Low-Dose-Rate Brachytherapy. Int J Radiat Oncol 2014;88(3):715-22. https://doi. org/10.1016/j.ijrobp.2013.11.241.

[26] Lyman JT. Complication probability as assessed from dose-volume histograms. Radiat Res Suppl 1985;8:S13-9. https://doi.org/10.2307/3583506.

[27] Gerald JKCB. Calculation of complication probability factors for non-uniform normal tissue irradiation: The effective volume method. Int J Radiat Oncol Biol Phys 1989;16:1623-30.

[28] Burman C, Kutcher GJ, Emami B, Goitein M. Fitting of normal tissue tolerance data to an analytic function. Int J Radiat Oncol Biol Phys 1991;21(1):123-35. https:// doi.org/10.1016/0360-3016(91)90172-Z.

[29] Niemierko a. Reporting and analyzing dose distributions: a concept of equivalent uniform dose. Med Phys 1997;24:103-10. https://doi.org/10.1118/1.598154.

[30] Fukahori M, Matsufuji N, Himukai T, Kanematsu N, Mizuno H, Fukumura A, et al. Estimation of late rectal normal tissue complication probability parameters in carbon ion therapy for prostate cancer. Radiother Oncol 2016;118(1):136-40. https://doi.org/10.1016/j.radonc.2015.11.023.

[31] Tucker SL, Dong L, Bosch WR, Michalski J, Winter K, Lee AK, et al. Fit of a Generalized Lyman Normal-Tissue Complication Probability (NTCP) Model to Grade $\geq 2$ Late Rectal Toxicity Data From Patients Treated on Protocol RTOG 94-06. Int J Radiat Oncol Biol Phys 2007;69:S8. https://doi.org/10.1016/j. ijrobp.2007.04.078.

[32] Söhn M, Yan D, Liang J, Meldolesi E, Vargas C, Alber M. Incidence of late rectal bleeding in high-dose conformal radiotherapy of prostate cancer using equivalent uniform dose-based and dose-volume-based normal tissue complication probability models. Int J Radiat Oncol Biol Phys 2007;67(4):1066-73. https://doi.org/ 10.1016/j.ijrobp.2006.10.014.

[33] Rancati T, Fiorino C, Gagliardi G, Cattaneo GM, Sanguineti G, Borca VC, et al Fitting late rectal bleeding data using different NTCP models: Results from an Italian multi-centric study (AIROPROS0101). Radiother Oncol 2004;73(1):21-32. https://doi.org/10.1016/j.radonc.2004.08.013.

[34] Gulliford SL, Partridge M, Sydes MR, Webb S, Evans PM, Dearnaley DP. Parameters for the Lyman Kutcher Burman (LKB) model of Normal Tissue Complication Probability (NTCP) for specific rectal complications observed in clinical practise. Radiother Oncol 2012;102(3):347-51. https://doi.org/10.1016/j. radonc.2011.10.022.

[35] Brenner DJ. Fractionation and late rectal toxicity. Int J Radiat Oncol Biol Phys 2004;60(4):1013-5. https://doi.org/10.1016/j.ijrobp.2004.04.014.

[36] Spratt DE, Pei X, Yamada J, Kollmeier MA, Cox B, Zelefsky MJ. Long-term survival and toxicity in patients treated with high-dose intensity modulated radiation therapy for localized prostate cancer. Int J Radiat Oncol Biol Phys 2013;85(3): 686-92. https://doi.org/10.1016/j.ijrobp.2012.05.023.

[37] Hoffman KE, Voong KR, Pugh TJ, Skinner H, Levy LB, Takiar V, et al. Risk of late toxicity in men receiving dose-escalated hypofractionated intensity modulated prostate radiation therapy: results from a randomized trial. Int J Radiat Oncol Biol Phys 2014;88(5):1074-84. https://doi.org/10.1016/j.ijrobp.2014.01.015.

[38] Kupelian PA, Thakkar VV, Khuntia D, Reddy CA, Klein EA, Mahadevan A Hypofractionated intensity-modulated radiotherapy (70 Gy at $2.5 \mathrm{~Gy}$ per fraction) for localized prostate cancer: Long-term outcomes. Int. J Radiat Oncol 2005;63(5): 1463-8. https://doi.org/10.1016/j.ijrobp.2005.05.054.

[39] Takemoto S, Shibamoto Y, Sugie C, Manabe Y, Yanagi T, Iwata H, et al. Long-term results of intensity-modulated radiotherapy with three dose-fractionation regimens for localized prostate cancer. J Radiat Res 2019; 60:221-7. Doi: 10.1093/jrr/ rry089.

[40] Patel N, Faria S, Cury F, David M, Duclos M, Shenouda G, et al. Hypofractionated radiation therapy ( 66 gy in 22 fractions at 3 gy per fraction) for favorable-risk prostate cancer: Long-term outcomes. Int J Radiat Oncol Biol Phys 2013;86(3): 534-9. https://doi.org/10.1016/j.ijrobp.2013.02.010.

[41] Ishikawa H, Tsuji H, Kamada T, Yanagi T, Mizoe J-E, Kanai T, et al. Carbon ion radiation therapy for prostate cancer: Results of a prospective phase II study. Radiother Oncol 2006;81(1):57-64. https://doi.org/10.1016/j. radonc.2006.08.015.

[42] Nomiya T, Tsuji H, Kawamura H, Ohno T, Toyama S, Shioyama Y, et al. A multiinstitutional analysis of prospective studies of carbon ion radiotherapy for prostate cancer: A report from the Japan Carbon ion Radiation Oncology Study Group (JCROS). Radiother Oncol 2016;121(2):288-93. https://doi.org/10.1016/j. radonc.2016.10.009.

[43] Brenner DJ, Hall EJ. Fractionation and protraction for radiotherapy of prostate carcinoma. Int J Radiat Oncol Biol Phys 1999;43(5):1095-101. https://doi.org/ 10.1016/S0360-3016(98)00438-6.

[44] Oliveira SM, Teixeira NJ, Fernandes L. What do we know about the $\alpha / \beta$ for prostate cancer? Med Phys 2012;39(6Part1):3189-201. https://doi.org/10.1118/ 1.4712224.

[45] Cosset JM, Chargari C, Créhange G. Which alpha/beta ratio for prostate cancer in 2019? Cancer/Radiotherapie 2019;23:342-5. https://doi.org/10.1016/j. canrad.2019.01.004. 